\title{
Observed Structural Heterogeneity of Human Hepatitis B Virus Surface Antigen Particles by Cryo-electron Microscopy
}

\author{
John R. Gallagher ${ }^{1}$, Audray K. Harris ${ }^{1}$ \\ ${ }^{1}$ Laboratory of Infectious Diseases, National Institute of Allergy and Infectious Diseases, National \\ Institutes of Health, Bethesda, MD, USA.
}

Due to the integrated use of bioinformatics, structural biology, and gene synthesis, the design of vaccine nanoparticles is an area of research that is gaining interest. This is due to the fact that conserved epitopes can be engineered to be displayed on nanoparticles with improved immunogenicity. One example of this antigen design concept is the use of ferritin to display epitopes from the influenza viral glycoprotein, hemagglutinin [1,2]. Ferritin is a particle with octahedral symmetry, and epitopes displayed on ferritin have been shown to elicit protective immune responses [1,2,3]. Interestingly, hepatitis B virus (HBV) surface antigen particles ( $\mathrm{sAg}$ ) isolated from sera were used as the first HBV vaccine and later replaced with recombinant sAg particles [4,5]. Furthermore, the sAg particles had been reported also to have octahedral symmetry by single-particle cryo-electron microscopy (cryo-EM) analysis [6]. To the contrary, tomographic analysis by cryo-EM did not detect octahedral symmetry [7]. Thus, whether nanoparticles that are efficacious in animals and humans must have strict octahedral has not been explored. To address the question of the structural organization of HBV sAg particles, we analyzed sAg particles of the Ad subtype isolated from human sera. We used the methods of single-particle cryo-EM with movie-mode data collection, resolution assessment, 2D classification and 3D reconstruction.

Particles isolated from HBV positive sera presented isolated particles when observed in cryo-EM images (Figure 1a). Images were acquired on a Titan Krios at $300 \mathrm{keV}$ with a Falcon II direct detector in movie mode to collect a series of frames for each image area (Figure 1a). The software MotionCor2 was used to align frames for each region of interest. The software CTFFind4 was used to assesses the resolution of the aligned images (Figure 1b). The majority of images had data at resolutions beyond $7 \AA$ (Figure $1 \mathrm{~b}$ ). The sAg is predicted to be an alpha helical protein. At such resolutions, secondary structural elements such as alpha-helices would be apparent for a symmetrical particle. However, analysis of 125,405 particles using RELION reference-free 2D classification with no symmetry imposed did not produce classes with apparent 2-fold, 3-fold, and 4-fold views that would be expected for particles with octahedral symmetry [2] (Figure 1c). However, the particles did appear to have two density layers, with the outer layer having protruding surface spikes. The diameters were in the range of 20 to $25 \mathrm{~nm}$. The use of octahedral or icosahedral symmetry did not produce structures with subnanometer resolution and hence no discernable alpha helical structure. To further analyze the sAg particle, we selected the subset of particles that were $22 \mathrm{~nm}$ in diameter $(29,276$ particles) for asymmetric reconstruction and found that the resolution was limited to about $20 \AA$ (Figure 2a). Examination of the reconstruction showed an asymmetric structure with protruding spikes (Figure $2 \mathrm{~b}$ ). Slices through the 3D reconstruction revealed a spiky surface with a double layered architecture with an apparent low-density or empty center (Figure 1c). Our results suggest that human sAg particles might not have strict symmetry to which the consitutent sAg molecules are organized. This suggests that vaccine nanoparticles could deviate from rigid symmetry and still be effective immunogens. Whether such structural heterogeneity improves immunogenicity is something that can be explored within further structure-guided design efforts to create vaccine nanoparticles as immunogens for infectious disease agents. 
References:

[1] Kanekiyo, M. et al. Nature, 499 (2013), p.102.

[2] Yassine, H.M. et al. Nat. Med., 21 (2015), p.1065.

[3] Kanekiyo, M. et al. Cell, 162 (2015), p.1090.

[4] He, L. et al. Nat. Commun., 7 (2016), p.1.

[5] Zuckerman, A.J. Lancet, 1 (1979), p. 547.

[6] Gilbert, R.J. et al. Proc. Natl. Acad. Sci. USA, 102 (2015) p. 14783.

[7] Gallagher, J.R. et al. Virology, 502 (2017) p. 176.

a

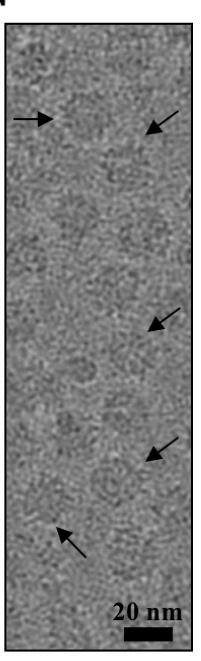

b

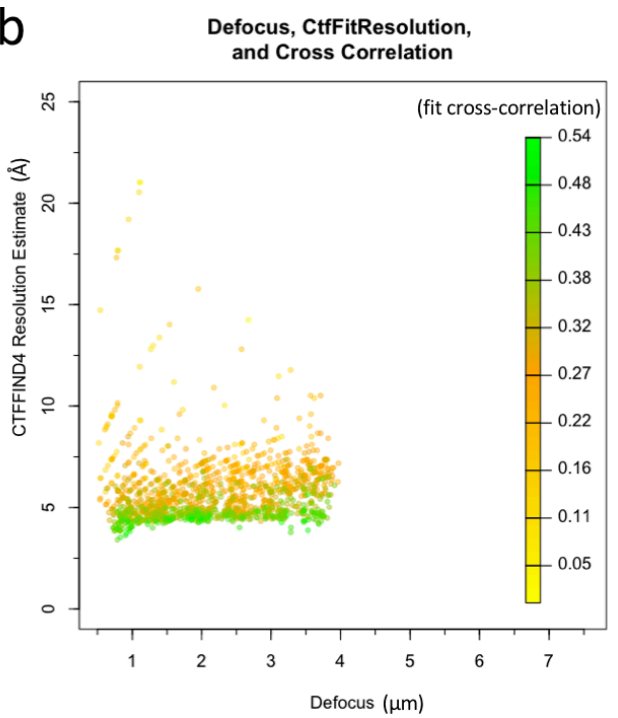

C

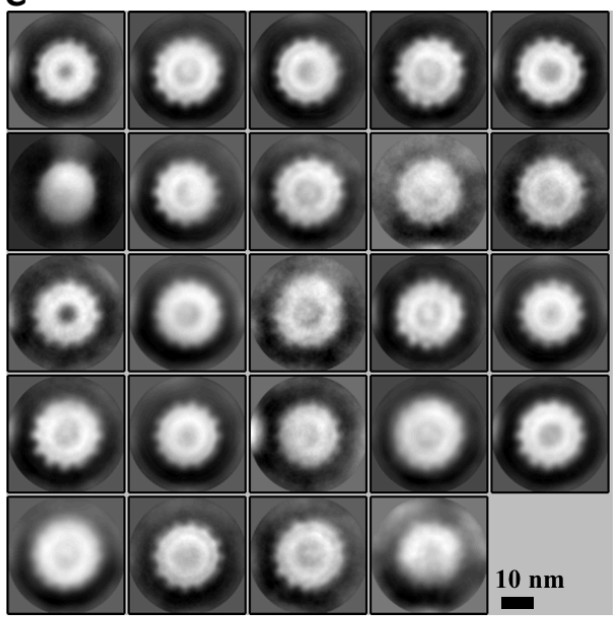

Figure 1. Analysis of surface antigen (sAg) particles. (a) cryo-EM of particles. (b) Resolution assessment of images by estimating potential resolution as a function of defocus in microns. (C) Reference-free 2D classes of sAg particles. No symmetry was imposed. Scale bars, $20 \mathrm{~nm}, 10 \mathrm{~nm}$.

a

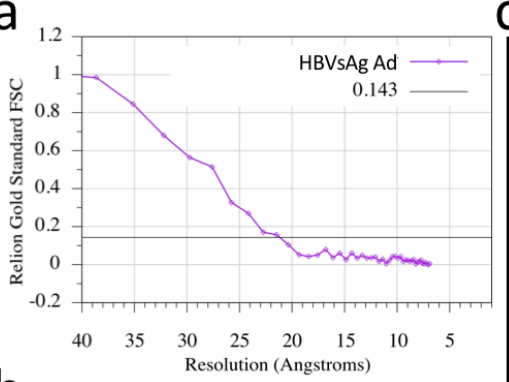

b

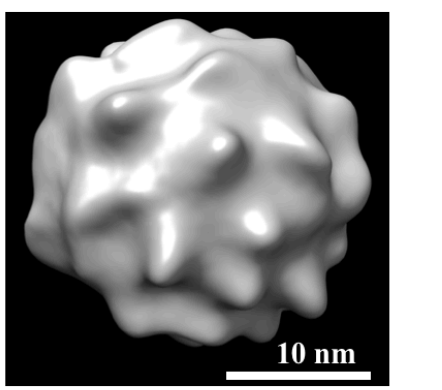

C

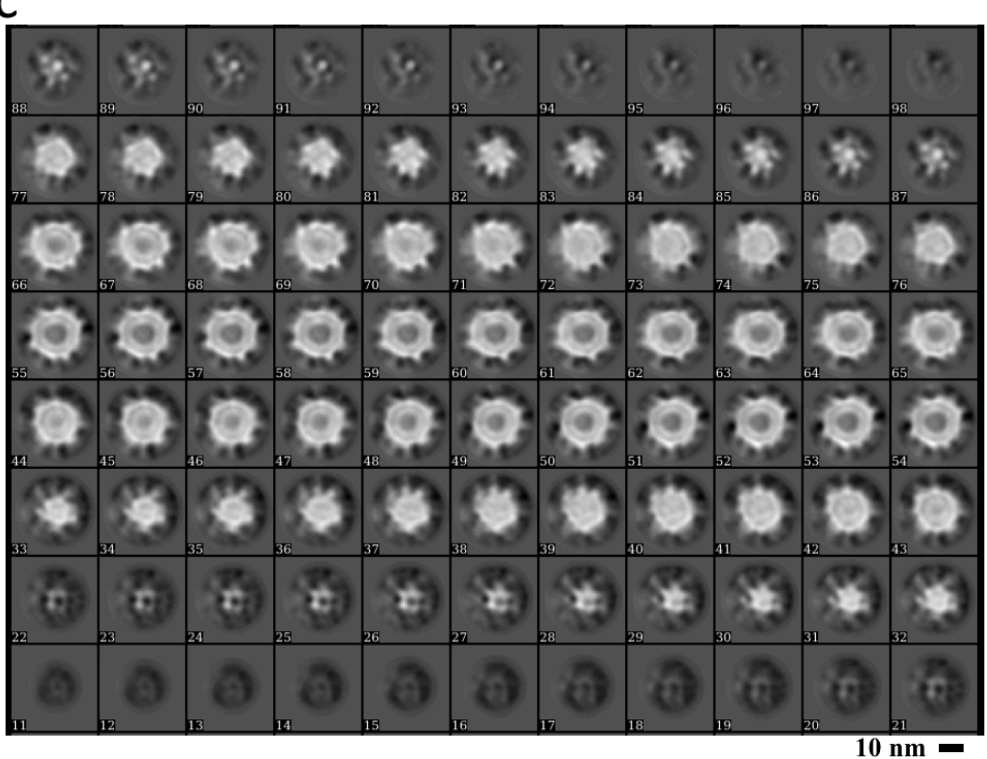

Figure 2. 3D reconstruction of sAg particle. (a) Resolution assessed by Fourier Shell Correlation (FSC cutoff $=0.143$ ). (b) Asymmetric reconstruction of $\mathrm{sAg}$ particles that were $22 \mathrm{~nm}$ in diameter. (c) Serial slices through the sAg 3D reconstruction. Scale bars, $10 \mathrm{~nm}$. 